\title{
Vertebrados terrestres registrados mediante foto-trampeo en arroyos estacionales y cañadas con agua superficial en un hábitat semiárido de Baja California Sur, México
}

\section{Terrestrial vertebrates recorded by camera traps in areas with seasonal streams and creeks of superficial waters in a semiarid habitat of Baja California Sur, Mexico}

\author{
Erika Mesa-Zavala, Sergio Álvarez-Cárdenas ${ }^{\bigotimes}$, Patricia Galina-Tessaro, Enrique Troyo-Diéguez e Israel \\ Guerrero-Cárdenas
}

Centro de Investigaciones Biológicas del Noroeste, S.C. Apartado postal 128, 23090 La Paz, Baja California Sur, México.

\salvarez04@cibnor.mx

\begin{abstract}
Resumen. Los cuerpos de agua superficial (CAS) permanentes o efímeros (pozas, tinajas, escurrimientos, etc.) que se encuentran en depresiones del terreno, como arroyos y cañadas, son soporte fundamental para el ecosistema en zonas áridas. Mediante el uso de cámaras-trampa, en este estudio se identifican especies de vertebrados terrestres silvestres presentes en 4 sitios con agua superficial, en el extremo sur de la sierra El Mechudo, Baja California Sur, y se analiza el uso de los CAS por las especies en los periodos de actividad. En cada sitio se caracterizó el hábitat (topografía, vegetación y agua). Los 4 sitios mostraron diferencias en sus características ambientales. Se identificaron 41 especies de vertebrados terrestres (3 reptiles, 31 aves y 7 mamíferos). Se encontraron también varias especies de murciélagos que no fueron identificadas. La riqueza de especies y frecuencia de visita fue diferente en cada sitio. Con excepción de 3 especies de mamíferos, el horario de actividad fue similar en los 4 sitios. La presente investigación aporta información sobre la importancia de los CAS en zonas semiáridas, describiendo el hábitat, las especies y su comportamiento, elementos básicos para la conservación y manejo de los recursos naturales.
\end{abstract}

Palabras clave: fauna, riqueza específica, cuerpos de agua superficial, sierra El Mechudo.

\begin{abstract}
Permanent or ephemeral water ponds (puddles, catchments, drains, and so on) located on ground depressions, such as streams and creeks, are a fundamental support for ecosystems in dry areas. This study identified the species of native terrestrial vertebrates in 4 sites in the southernmost part of the Sierra El Mechudo, B.C.S., including how such species use these bodies of water based on the periods of species activity. Habitats were characterized in 4 sites (topography, vegetation, and water sources); camera-traps were placed around water ponds from March to October 2007. The 4 sites differed in their environmental characteristics. Overall, there were 41 species of terrestrial vertebrates ( 3 reptiles, 31 birds, and 7 mammals), with several unidentified species of bats that were also found. Specific richness and visit (record) frequency were also different at each site. Species activity schedule was similar in the 4 sites with the exception of 3 species of mammals. This study provides important information of superficial water in semiarid areas, including description of habitat, species, and their behavior, which are basic elements for conservation and management of natural resources.
\end{abstract}

Key words: fauna, species richness, superficial water bodies, Sierra El Mechudo.

\section{Introducción}

En México, las zonas áridas abarcan la mayor parte del Altiplano Mexicano, la planicie costera de Sonora y la península de Baja California (Rzedowski, 1978; MacMahon, 1979; Briones, 1994). El patrón hidrológico-ambiental que predomina y caracteriza los desiertos es la escasez de agua, recurso que puede variar de manera espacial y tem-

Recibido: 10 diciembre 2010; aceptado: 24 junio 2011 poral (Whitford, 2002; Rosenstock et al., 2005). El recurso hídrico constituye un factor limitante para las poblaciones de fauna silvestre en los ambientes desérticos (Broyles, 1995; Rosenstock et al., 2005). De esta manera, los cuerpos de agua superficial (CAS), permanentes o efímeros (pozas, tinajas, lloraderos etc.), afectan la distribución y abundancia de plantas y animales, aún cuando numerosas especies de vertebrados terrestres tienen la capacidad de obtener agua de diferentes maneras, ya sea bebiéndola directamente, ingiriéndola en su alimento o por oxidación del material alimenticio (Nagy, 1975; Turner y Weaver, 
1980; Broyles y Cutler, 1999; Krausman et al., 2006). Por consiguiente, el agua constituye uno de los principales factores que determinan la presencia y el uso de hábitat de las especies (Shmida et al., 1986; Broyles, 1995; Rosenstock et al., 2005), en particular cuando está restringido o limitado para algunas especies, como Odocoileus hemionus y Ovis canadensis (Jaramillo-Monroy et al., 1991; Turner et al., 2004; Marshal et al., 2006).

Se han realizado diversos estudios de hábitat, conductuales y fisiológicos sobre fauna que habita en zonas áridas, relacionados con el agua superficial; así, Krausman et al. (2006) recopilaron varios trabajos vinculados al uso de los CAS por la fauna, encontrando que los atributos del hábitat que rodean las fuentes de agua, como la cobertura vegetal, la calidad del forraje, la topografía y la distancia entre las fuentes de agua afectan el uso de los mismos. Como parte de un estudio sobre $O$. canadensis y su hábitat, el presente trabajo surge con la finalidad de registrar las especies de vertebrados terrestres presentes en 4 sitios con CAS, permanentes o temporales del extremo sur de la sierra El Mechudo, Baja California Sur, así como del uso de estos cuerpos, con base en los periodos de actividad de las especies, registradas mediante cámaras-trampa.

\section{Materiales y métodos}

Área de estudio. La presente investigación se realizó en 4 sitios ubicados en el extremo sur de la sierra El Mechudo, en Baja California Sur, México, entre las coordenadas $24^{\circ} 16^{\prime}-24^{\circ} 32^{\prime} \mathrm{N}, 110^{\circ} 38^{\prime}-110^{\circ} 50^{\prime} \mathrm{O}$ (Fig. 1). La sierra está formada por diferentes cadenas montañosas paralelas a la costa, cuyas laderas orientales son pronunciadas y dirigidas hacia el golfo de California formando numerosos cañones, acantilados y lechos de arroyos, mientras que las laderas occidentales descienden gradualmente hacia el océano Pacífico. La elevación promedio de esta sierra es de $550 \mathrm{~m}$ snm y la máxima de $1070 \mathrm{~m} \mathrm{snm}$ en el cerro El Mechudo (Álvarez-Cárdenas et al., 2001).

El clima es seco, con temperatura media anual mayor de $22^{\circ} \mathrm{C}$, régimen de lluvias de verano, porcentaje de lluvia invernal de 5 a $10.2 \%$ del total anual (García, 1973) y periodos sin precipitación que pueden durar varios años (León de la Luz et al., 2008). Con base en la información de la estación meteorológica Alfredo V. Bonfil (la más cercana al área de estudio), en la figura 2 se muestra el diagrama de temperatura media y precipitación total. La temperatura media anual es de $23.7^{\circ} \mathrm{C}$, la media máxima de $29.4^{\circ} \mathrm{C}$ y la mínima media de $18.0^{\circ} \mathrm{C}$. La precipitación anual promedio es de $140 \mathrm{~mm}$ y se presenta en 2 periodos; el más intenso en agosto y septiembre (incidencia de huracanes), el otro, en diciembre y enero, corresponde a la lluvia invernal. La temporada seca es de marzo a junio (Fig. 2).

En la sierra El Mechudo, el agua libre se encuentra en diferentes tipos de CAS, incluyendo depresiones, tinajas, ojos de agua, oquedades entre las rocas y en arroyos intermitentes en un número importante de cañones y cañadas que cruzan la sierra hacia el golfo de California. Algunos de los CAS pueden permanecer todo el año (ÁlvarezCárdenas et al., 2001; León de la Luz et al., 2008); sin embargo, no existe estimación del número ni datos sobre su ubicación. El matorral sarcocaule es la vegetación dominante, se caracteriza por leguminosas, suculentas y semisuculentas. En arroyos, cañadas o fondos de cañón, la vegetación xero-riparia aparece en forma esporádica (León de la Luz et al., 2008); en algunos de ellos destaca la presencia de palmares.

Caracterización del hábitat. En 4 localidades, La Cañada Norte, El Saucito, El Camarón y El Sauzoso, se registró la topoforma (cañón, cañada y arroyo), la altura e inclinación de sus laderas, distancia entre las laderas (amplitud) y elevación. La caracterización de la vegetación riparia se realizó por medio de transectos de dimensiones fijas de 50 metros de largo por 10 de ancho, distribuidos a lo largo de estos sitios; el número de transectos realizados dependió del área de cada sitio. En cada transecto se identificaron las especies vegetales y el número de individuos de cada una; se midió la altura y la cobertura del dosel (ancho y largo) de cada individuo.

Para establecer la cantidad de agua disponible se determinó el volumen de todos los CAS en cada sitio, considerando el largo, ancho y profundidad media.

Presencia de vertebrados. Los registros de anfibios, reptiles, aves y mamíferos silvestres se realizaron de marzo a octubre de 2007, utilizando cámaras-trampa automáticas (cámaras digitales con sensor infrarrojo de movimiento, marca Moultrie, con resolución de imagen de 2.1 MP). Cada cámara-trampa fue programada para tomar 2 fotografías por cada detección, con intervalo de 60 segundos. Cada mes se colocaron 5 cámaras-trampa durante una semana en cada una de las 4 áreas, ubicándolas en sitios con agua disponible, los cuales fueron seleccionados por la visibilidad y seguridad de las cámaras-trampa, de manera que abarcaran los sitios de probable acceso de los animales; en ningún caso fueron cebadas las cámaras-trampa con alimento o atrayentes de olor.

Para determinar el esfuerzo de muestreo se cuantificaron las cámaras-trampa activas por día, y el número de fotografías con fauna presente en cada sitio. La frecuencia de visita de cada especie se consideró como el número de organismos que se presentaron en las fotografías durante el mismo lapso. Se registró el número de $\mathrm{CAS}$ en los que se presentó cada especie por día, diferenciando las especies más comunes o registradas en mayor cantidad de CAS y 


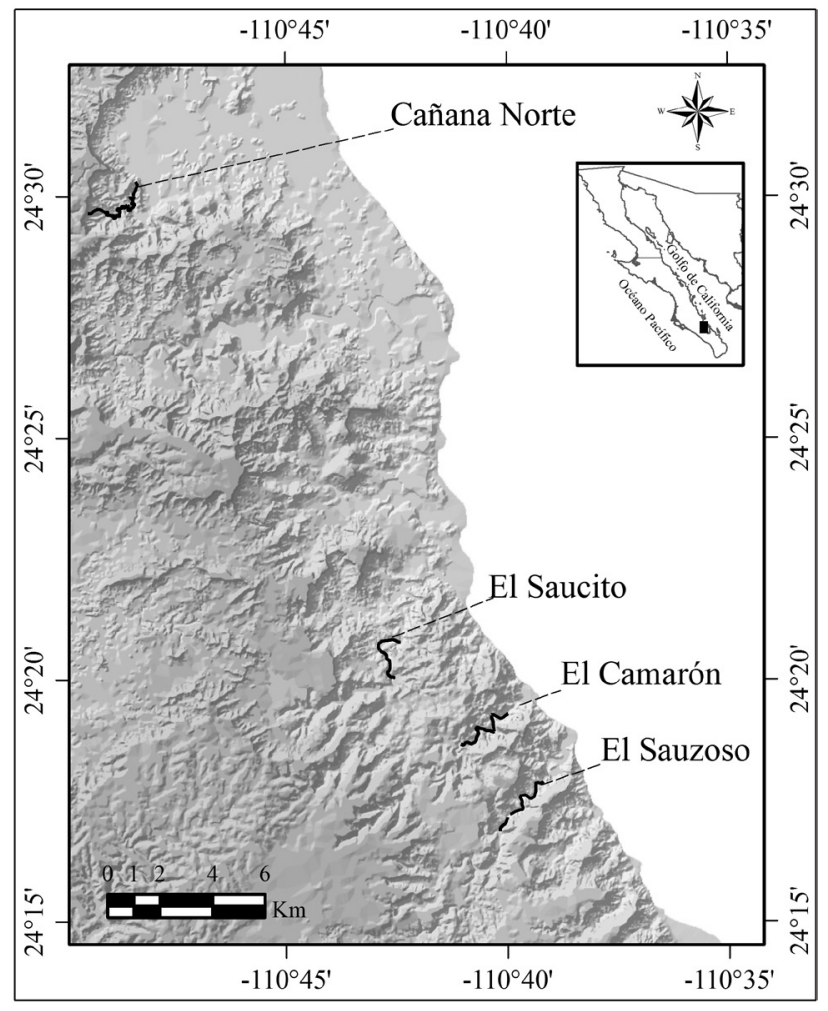

Figura 1. Ubicación de los sitios estudiados: Cañada Norte, El Saucito, El Camarón y El Sauzoso, en el sur de la sierra El Mechudo, Baja California Sur.

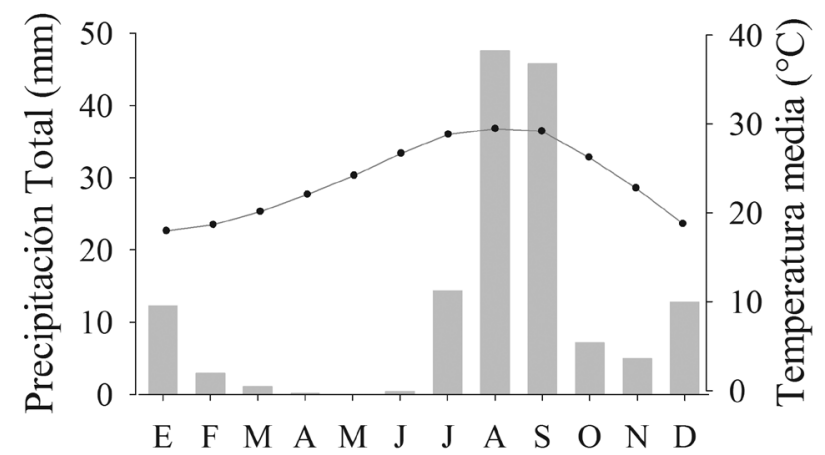

Figura 2. Diagrama de precipitación total (barra gris) y temperatura media (punto negro) (1980-2007) para el sur de la sierra El Mechudo, Baja California Sur.

las especies menos comunes o con escasos registros en los CAS.

Uso de cuerpos de agua superficial. El uso de los CAS se evaluó en función de los horarios de actividad de cada especie. Se consideraron (a) diurnas, las fotografías con luz de día, sin el flash activado y (b) nocturnas, las tomadas en completa oscuridad, con el flash activo. En el caso de las especies que se presentaron tanto en el día como en la noche se obtuvo la proporción de cada horario.
Para examinar la separación de las especies respecto al horario de actividad y la frecuencia de visita se aplicó el análisis de componentes principales (ACP) (STATISTICA 8.0, Statsoft Inc, Tulsa, Oklahoma). Los componentes principales que resultan con eigenvalores mayores a 1.0 se consideraron estadísticamente significativos (Kaiser, 1960).

\section{Resultados}

Caracterización del hábitat. El área muestreada para caracterizar la topografía y la vegetación en cada sitio varió de acuerdo con el tamaño del mismo; en El Camarón fue de $3000 \mathrm{~m}^{2}$, en El Sauzoso y El Saucito fue de $2000 \mathrm{~m}^{2}$ y en la Cañada Norte de $1000 \mathrm{~m}^{2}$.

a) El Sauzoso $\left(24^{\circ} 17^{\prime} \mathrm{N}, 110^{\circ} 40^{\prime} \mathrm{O}\right)$ es un arroyo amplio, cuya ladera oriental presenta una pared vertical de aproximadamente $40 \mathrm{~m}$, en tanto que la ladera opuesta presenta pendientes menores a $45^{\circ}$. La amplitud máxima del arroyo es de $60 \mathrm{~m}$ y la elevación de los CAS muestreados varía de 51 a $65 \mathrm{~m}$ snm. Se identificaron 27 especies vegetales, con una abundancia total de 152 individuos, una densidad de 0.076 ind $/ \mathrm{m}^{2}$, cobertura del dosel de $34.40 \%$ y altura promedio de $1.69 \mathrm{~m}$. La especie dominante fue el mezquite Prosopis articulata, y la asociación principal $P$. articulata-binorama Acacia farnesiana.

b) El Camarón $\left(24^{\circ} 19^{\prime} \mathrm{N}, 110^{\circ} 40^{\prime} \mathrm{O}\right)$ tiene características de arroyo y cañón con laderas orientadas este-oeste; la distancia entre las paredes, que no son muy elevadas, es de 30 a $50 \mathrm{~m}$ y se va estrechando hacia el fondo hasta perderse en un cerro rocoso. En varias partes presenta fracturas topográficas (vínculo con diferentes cañadas y cerros). Tiene una variación de 44 a $49 \mathrm{~m}$ snm entre los CAS localizados. Se encontraron 33 especies de plantas con una abundancia de 152 individuos, densidad de $0.050 \mathrm{ind} / \mathrm{m}^{2}$, cobertura de $35.23 \%$ y altura promedio de $2.64 \mathrm{~m}$, lo que evidencia la mayor altura promedio de la vegetación de los 4 sitios. En este arroyo se encontró la principal asociación de palo blanco-palo santo, Lysiloma candida-Viscainoa geniculata, en la cual domina la primera especie. A lo largo del cañón se distribuyen 3 palmares de Washingtonia robusta.

c) El Saucito $\left(24^{\circ} 20^{\prime} \mathrm{N}, 110^{\circ} 42^{\prime} \mathrm{O}\right)$ presenta características topográficas entre arroyo y cañón con paredes pronunciadas y orientación este-oeste que varían entre 20 y $60 \mathrm{~m}$ de altura; la separación entre las paredes es de 25 a 35 m, con elevación mínima de 59 m snm y máxima de $107 \mathrm{~m}$ snm, entre los sitios de muestreo. En El Saucito se identificó la mayor riqueza específica y abundancia de los 4 sitios, con 46 especies y 234 individuos respectivamente; presentó una densidad de $0.117 \mathrm{ind} / \mathrm{m}^{2}, 38.04 \%$ de cobertura y altura promedio de $1.90 \mathrm{~m}$. La especie dominante fue $L$. candida, que 
mostró asociación con el teso, Acacia greggii. Se detectaron algunos individuos de palmera $W$. robusta.

d) La Cañada Norte $\left(24^{\circ} 29^{\prime} \mathrm{N}, 110^{\circ} 48^{\prime} \mathrm{O}\right)$ tiene propiedades físicas similares a un fondo de cañón; en una ladera presenta paredes con altura aproximada de $40 \mathrm{~m}$, mientras que la otra tiene inclinación mayor de 45 grados; el ancho máximo es alrededor de $10 \mathrm{~m}$; la elevación difiere entre 50 y $81 \mathrm{~m}$ snm. Esta cañada es el menor de los 4 sitios, con una riqueza específica de 19 y una abundancia total de 204 individuos; sin embargo, de los 4 sitios es el que presentó la mayor densidad y cobertura con $0.204 \mathrm{ind} / \mathrm{m}^{2}$ y $83.28 \%$ respectivamente, y $2.27 \mathrm{~m}$ de altura promedio. En este sitio, la especie dominante fue el incienso, Encelia farinosa, asociada con la jojoba, Simmondsia chinensis.

La cantidad aproximada de agua superficial dentro de cada sistema varió notoriamente entre los sitios. El Sauzoso $\left(619.17 \mathrm{~m}^{3}\right)$ fue el que mayor volumen de agua tuvo, seguido de El Camarón $\left(55.79 \mathrm{~m}^{3}\right)$, El Saucito $\left(50.62 \mathrm{~m}^{3}\right)$ y la Cañada Norte $\left(0.32 \mathrm{~m}^{3}\right)$.

Presencia de vertebrados. El registro de fauna en el área de estudio se realizó en un total de 338 días/cámara, variando el número de cámaras activas por sitio y el tiempo de permanencia, ello a consecuencia de dificultades técnicas en el uso de los equipos y a cambios en las condiciones climáticas. Los días de uso de equipo para cada sitio fueron: El Sauzoso 77, El Camarón 83, El Saucito 107 y la Cañada Norte 71 días/cámara. El número de fotografías con animales presentes varió también entre los sitios: El Sauzoso 925, El Camarón 770, El Saucito 458 y la Cañada Norte 89 (Fig. 3).

La riqueza de especies registradas mediante el uso de las cámaras-trampa fue de 41 ( 3 reptiles, 31 aves y 7 mamíferos, pertenecientes a 2, 22 y 6 familias, respectivamente; por la dificultad para identificarlas en las fotografías, todas las especies de murciélagos se consideraron como un grupo). De las especies registradas en el presente estudio, 4 se encuentran listadas en la Norma Oficial Mexicana-059 (SEMARNAT, 2010). En la categoría de amenazadas (A), están Callisaurus draconoides, Taxidea taxus y Urosaurus nigricaudus y en la de protección especial (Pr), O. canadensis. En el Cuadro 1 se observa la riqueza de especies en cada sitio de acuerdo con cada grupo taxonómico, así como la frecuencia de visita, lo cual muestra que las aves y mamíferos fueron los grupos mejor representados. La Cañada Norte es el sitio que presentó menor riqueza de especies y frecuencia de visita, mientras que El Camarón presentó la mayor riqueza específica y frecuencia de visita.

En la figura 4 se muestra el número de CAS en los que fue registrada cada especie. Se observa que hubo numerosas especies registradas 1 o 2 veces (16 y 9 especies, respectivamente), las cuales se consideraron como poco comunes durante este estudio. Por otra parte, las 6 especies más comunes se registraron en más de 10 CAS. Las especies que se registraron en mayor número de CAS fueron los murciélagos (39), Cathartes aura (14), Carpodacus mexicanus (13), Canis latrans (13), Zenaida asiatica (12) y $O$. canadensis (11). En el Cuadro 2 se listan las especies registradas en cada sitio.

En El Sauzoso, las especies más frecuentes de cada grupo taxonómico fueron $C$. draconoides (45 eventos), Callipepla californica (22) y los murciélagos (73). En El Camarón, la mayor frecuencia fue de C. draconoides (34), C. mexicanus (35) y los murciélagos (82). En El Saucito, la mayor frecuencia se dio en el grupo de las aves con $C$. aura (67) y en el de los mamíferos con Procyon lotor (13) y los murciélagos (13). En la Cañada Norte tuvieron mayor frecuencia Columbina passerina (18) y O. canadensis (8). El Sauzoso fue el único sitio con registro de Aspidoscelis tigris, Actitis macularia, C. californica, Chordeiles acutipennis, Pheucticus melanocephalus, Lynx rufus y T. taxus (Cuadro 2).

En El Camarón se registró con exclusividad $U$. nigricaudus, Bubo virginianus, Campylorhynchus brunneicapillus, Picoides scalaris, Polioptila caerulea y Tachycineta thalassina (Cuadro 2). El Saucito fue el único sitio donde se registró Buteo jamaicensis, Falco sparverius, Megaceryle alcyon y Polioptila californica (Cuadro 2). En la Cañada Norte, la única especie presente fue Ammospermophilus leucurus, que no se registró en otros sitios (Cuadro 2).

Uso de cuerpos de agua superficial. Durante este estudio se registraron 35 especies activas en el horario diurno, 4 en el nocturno (contabilizando los murciélagos como

Cuadro 1. Riqueza específica y frecuencia de visita (entre paréntesis) de los grupos taxonómicos de vertebrados terrestres registrados por medio de cámaras-trampa en los cuerpos de agua superficial en el sur de la sierra El Mechudo, Baja California Sur

\begin{tabular}{lcccc}
\hline Sitio & Reptiles & Aves & Mamiferos & Total \\
\hline El Sauzoso & $2(46)$ & $17(94)$ & $7(91)$ & $26(231)$ \\
El Camarón & $2(35)$ & $22(163)$ & $5(126)$ & $29(324)$ \\
El Saucito & $1(9)$ & $16(156)$ & $4(39)$ & $21(204)$ \\
Cañada Norte & $1(1)$ & $3(24)$ & $3(14)$ & $7(39)$ \\
Total & $3(91)$ & $31(437)$ & $8(270)$ & $42(798)$ \\
\hline
\end{tabular}




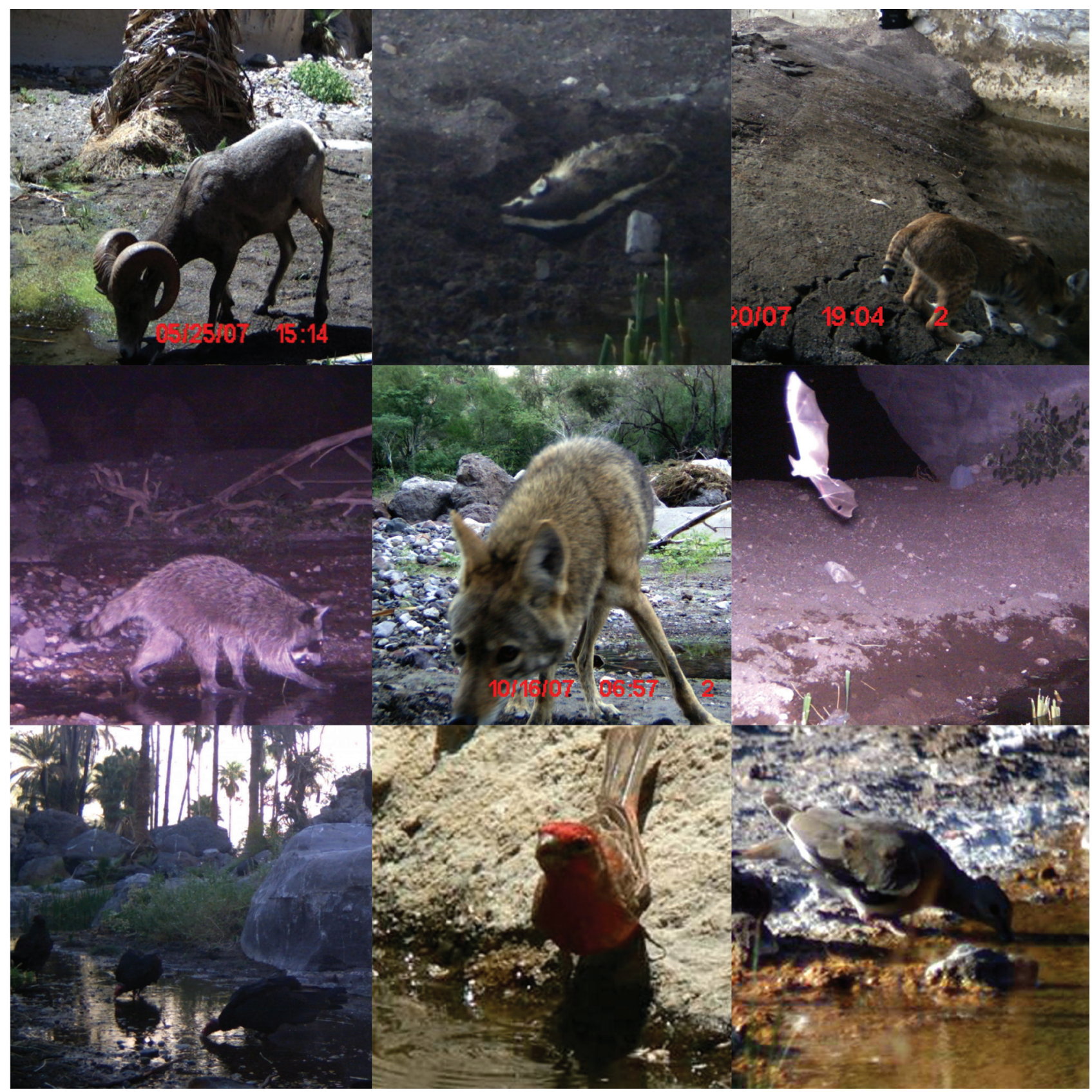

Figura 3. Fotografías de especies registradas por cámaras-trampa. De izquierda a derecha, borrego cimarrón, Ovis canadensis; tejón, Taxidea taxus; gato montés, Lynx rufus; mapache, Procyon lotor; coyote, Canis latrans; murciélago, aura, Cathartes aura; gorrión, Carpodacus mexicanus; paloma de alas blancas, Zenaida asiatica.

una especie) y 3 activas tanto en el día como en la noche (Cuadro 2). Las 3 especies de reptiles se registraron con actividad diurna. De las aves, en el horario diurno se presentaron 28 especies y 3 en el nocturno (que generalmente son de hábitos crepusculares-nocturnos). De los mamíferos, 4 especies estuvieron activas en el horario diurno, 3 se presentaron tanto en el día como en la noche y los murciélagos en el horario nocturno.
La proporción de cada una de las especies que se encontraron activas en los CAS tanto en el día como en la noche y el sitio donde se presentaron se especifican en el Cuadro 3. Se observa que $C$. latrans estuvo presente en mayor proporción durante el día en todos los sitios, opuesto a lo que sucede con $P$. lotor, que presentó mayor actividad en la noche, en los 3 sitios donde se registró. En el caso de Urocyon cinereoargenteus, su actividad en El Sauzoso sólo se 


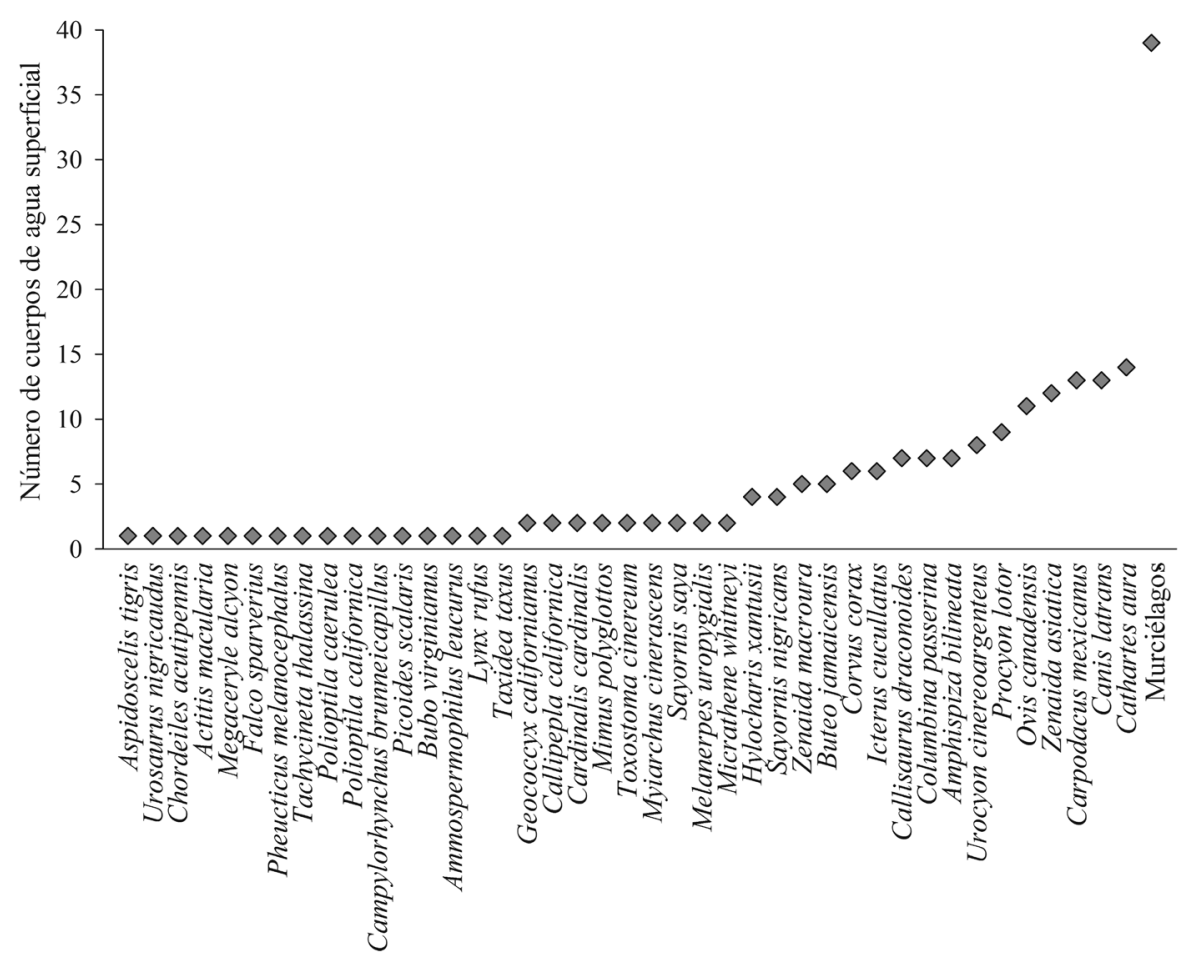

Figura 4. Número de cuerpos de agua superficial en los que se registró cada especie durante el tiempo de estudio, en el sur de la sierra El Mechudo, Baja California Sur.

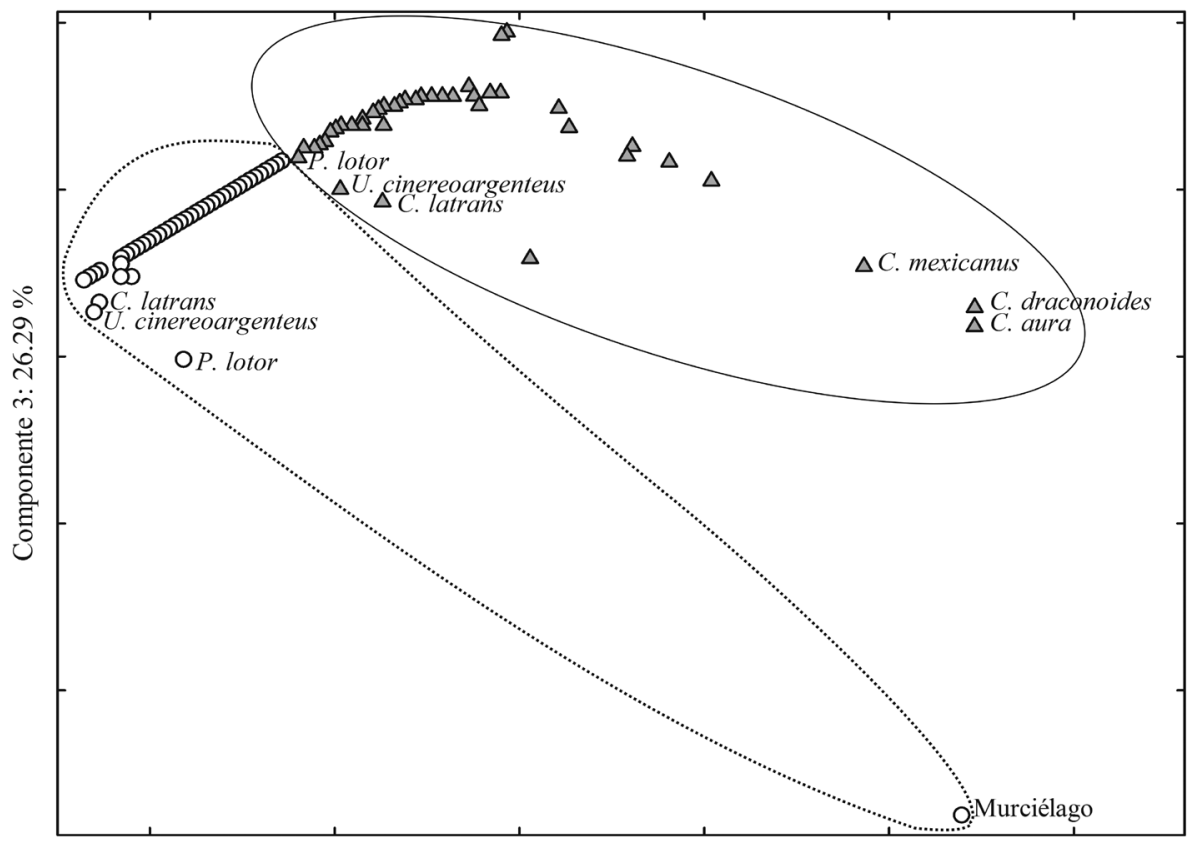

Componente 1: $40.38 \%$

Figura 5. Proyección de los casos del componente 1 y el componente 3 del análisis de componentes principales de las variables: especies, horario de actividad y frecuencia de visita a los cuerpos de agua superficial en la sierra El Mechudo, Baja California Sur. Grupo de especies con principal horario de actividad diurno (triángulo), grupo de especies con principal horario de actividad nocturno (círculo). 
Cuadro 2. Frecuencia de especies de vertebrados terrestres registrados por medio de cámaras-trampa en los cuerpos de agua superficial en 4 sitios del sur de la sierra El Mechudo, Baja California Sur, México

Frecuencia de visita

\begin{tabular}{|c|c|c|c|c|c|c|c|}
\hline $\begin{array}{c}\text { Grupo } \\
\text { taxonómico }\end{array}$ & Nombre cientifico & Nombre común & 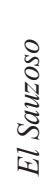 & 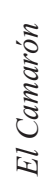 & 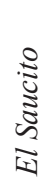 & 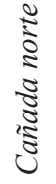 & $\begin{array}{c}\text { Horario de } \\
\text { actividad }\end{array}$ \\
\hline $\mathrm{Re}$ & Aspidoscelis tigris & Huico & 1 & 0 & 0 & 0 & $\mathrm{D}$ \\
\hline $\mathrm{Re}$ & Callisaurus draconoides & Lagartija arenera & 45 & 34 & 9 & 1 & $\mathrm{D}$ \\
\hline $\mathrm{Re}$ & Urosaurus nigricaudus & Lagartija de árbol & 0 & 1 & 0 & 0 & $\mathrm{D}$ \\
\hline Av & Hylocharis xantusii & Colibrí de Xantus & 0 & 3 & 3 & 0 & $\mathrm{D}$ \\
\hline $\mathrm{Av}$ & Chordeiles acutipennis & Tapacaminos & 4 & 0 & 0 & 0 & $\mathrm{~N}$ \\
\hline Av & Actitis macularia & Playerito manchado & 1 & 0 & 0 & 0 & $\mathrm{D}$ \\
\hline $\mathrm{Av}$ & Cathartes aura & Aura & 3 & 22 & 67 & 0 & $\mathrm{D}$ \\
\hline $\mathrm{Av}$ & Columbina passerina & Tortolita & 3 & 11 & 0 & 18 & $\mathrm{D}$ \\
\hline Av & Zenaida asiatica & Paloma alas blancas & 16 & 20 & 4 & 4 & $\mathrm{D}$ \\
\hline $\mathrm{Av}$ & Zenaida macroura & Paloma huilota & 1 & 8 & 1 & 0 & $\mathrm{D}$ \\
\hline Av & Megaceryle alcyon & Martín pescador & 0 & 0 & 1 & 0 & $\mathrm{D}$ \\
\hline $\mathrm{Av}$ & Geococcyx californianus & Correcaminos & 1 & 0 & 0 & 2 & $\mathrm{D}$ \\
\hline Av & Buteo jamaicensis & Gavilán cola roja & 0 & 0 & 6 & 0 & $\mathrm{D}$ \\
\hline Av & Falco sparverius & Cernícalo americano & 0 & 0 & 1 & 0 & $\mathrm{D}$ \\
\hline Av & Callipepla californica & Codorniz & 22 & 0 & 0 & 0 & $\mathrm{D}$ \\
\hline Av & Cardinalis cardinalis & Cardenal & 1 & 1 & 0 & 0 & $\mathrm{D}$ \\
\hline $\mathrm{Av}$ & Pheucticus melanocephalus & Picogordo & 4 & 0 & 0 & 0 & $\mathrm{D}$ \\
\hline Av & Corvus corax & Cuervo & 2 & 23 & 12 & 0 & $\mathrm{D}$ \\
\hline Av & Amphispiza bilineata & Gorrión garganta negra & 14 & 9 & 8 & 0 & $\mathrm{D}$ \\
\hline $\mathrm{Av}$ & Carpodacus mexicanus & Gorrión & 2 & 35 & 35 & 0 & $\mathrm{D}$ \\
\hline Av & Tachycineta thalassina & Golondrina verdemar & 0 & 2 & 0 & 0 & $\mathrm{D}$ \\
\hline $\mathrm{Av}$ & Icterus cucullatus & Calandria & 2 & 9 & 7 & 0 & $\mathrm{D}$ \\
\hline Av & Mimus polyglottos & Cenzontle & 2 & 1 & 0 & 0 & $\mathrm{D}$ \\
\hline Av & Toxostoma cinereum & Cuitlacoche peninsular & 8 & 1 & 0 & 0 & $\mathrm{D}$ \\
\hline $\mathrm{Av}$ & Polioptila caerulea & Perlita azulgris & 0 & 2 & 0 & 0 & $\mathrm{D}$ \\
\hline $\mathrm{Av}$ & Polioptila californica & Perlita californiana & 0 & 0 & 1 & 0 & $\mathrm{D}$ \\
\hline Av & Campylorhynchus brunneicapillus & Matraca del desierto & 0 & 4 & 0 & 0 & $\mathrm{D}$ \\
\hline Av & Myiarchus cinerascens & Papamoscas cenizo & 8 & 2 & 0 & 0 & $\mathrm{D}$ \\
\hline Av & Sayornis nigricans & Papamoscas negro & 0 & 2 & 3 & 0 & $\mathrm{D}$ \\
\hline Av & Sayornis saya & Mosquero llanero & 0 & 1 & 1 & 0 & $\mathrm{D}$ \\
\hline $\mathrm{Av}$ & Melanerpes uropygialis & Carpintero del desierto & 0 & 4 & 4 & 0 & $\mathrm{D}$ \\
\hline Av & Picoides scalaris & Carpintero mexicano & 0 & 1 & 0 & 0 & $\mathrm{D}$ \\
\hline Av & Bubo virginianus & Búho cornudo & 0 & 1 & 0 & 0 & $\mathrm{~N}$ \\
\hline Av & Micrathene whitneyi & Tecolote enano & 0 & 1 & 2 & 0 & $\mathrm{~N}$ \\
\hline $\mathrm{Ma}$ & Ammospermophilus leucurus & Juancito & 0 & 0 & 0 & 1 & $\mathrm{D}$ \\
\hline $\mathrm{Ma}$ & Ovis canadensis & Borrego cimarrón & 1 & 30 & 0 & 8 & $\mathrm{D}$ \\
\hline $\mathrm{Ma}$ & Canis latrans & Coyote & 7 & 3 & 11 & 0 & $\mathrm{D}-\mathrm{N}$ \\
\hline $\mathrm{Ma}$ & Urocyon cinereoargenteus & Zorra & 1 & 8 & 2 & 5 & $\mathrm{D}-\mathrm{N}$ \\
\hline $\mathrm{Ma}$ & Lynx rufus & Gato montés & 1 & 0 & 0 & 0 & $\mathrm{D}$ \\
\hline $\mathrm{Ma}$ & Taxidea taxus & Tejón & 1 & 0 & 0 & 0 & $\mathrm{D}$ \\
\hline $\mathrm{Ma}$ & Procyon lotor & Mapache & 7 & 3 & 13 & 0 & $\mathrm{D}-\mathrm{N}$ \\
\hline $\mathrm{Ma}$ & Murciélagos & Murciélagos & 73 & 82 & 13 & 0 & $\mathrm{~N}$ \\
\hline
\end{tabular}

Re, Reptilia; Av, Ave; Ma, Mammalia; D, registro diurno; N, registro nocturno. 
Cuadro 3. Proporción del horario de actividad de las especies diurnas-nocturnas registradas por medio de cámaras-trampa en los cuerpos de agua superficial en la sierra El Mechudo, Baja California Sur, México

\begin{tabular}{|c|c|c|c|c|c|c|}
\hline & \multicolumn{2}{|c|}{ Canis latrans } & \multicolumn{2}{|c|}{ Procyon lotor } & \multicolumn{2}{|c|}{ Urocyon cinereoargenteus } \\
\hline Sitio & $D$ & $N$ & $D$ & $N$ & $D$ & $N$ \\
\hline El Sauzoso & 0.9 & 0.1 & 0 & 1.0 & 0 & 1.0 \\
\hline El Camarón & 0.7 & 0.3 & 0.3 & 0.7 & 0.6 & 0.4 \\
\hline El Saucito & 0.7 & 0.3 & 0 & 1.0 & 0.5 & 0.5 \\
\hline Cañada Norte & -- & -- & -- & -- & 0.8 & 0.2 \\
\hline
\end{tabular}

$\mathrm{D}$, registro diurno; $\mathrm{N}$, registro nocturno.

Cuadro 4. Peso de los componentes principales para cada variable (especie, horario de actividad, frecuencia de visita) registrada en los cuerpos de agua superficial en la Sierra El Mechudo, Baja California Sur, Mexico

\begin{tabular}{lccc}
\hline & Componente 1 & Componente 2 & Componente 3 \\
\hline $\begin{array}{l}\text { Especie } \\
\text { Horario de }\end{array}$ & -0.002401 & -0.998610 & -0.052646 \\
$\begin{array}{l}\text { actividad } \\
\begin{array}{l}\text { Frecuencia } \\
\text { de visita }\end{array}\end{array}$ & -0.995768 & 0.002450 & -0.091870 \\
\hline
\end{tabular}

registró en la noche, en El Camarón y Cañada Norte realizó más visitas durante el día que en la noche y en El Saucito se registró en la misma proporción en el día y en la noche.

Mediante la aplicación del método ACP se obtuvieron 3 componentes principales; en el componente 1 la variable con mayor peso fue el horario de actividad; en el componente 2 , la especie y en el componente 3 , la frecuencia de visita a los CAS (Cuadro 4). Los eigenvalores de los componentes 1,2 y 3 fueron $1.21,1.00$ y 0.79 , en tanto que sus varianzas fueron $40.38,33.33$ y $26.29 \%$ respectivamente. Los componentes principales que son útiles son el 1 y 2 , debido a que son estadísticamente significativos (eigenvalor mayor a 1.0). Sin embargo, se graficó la dispersión de las especies con el componente 1 y el componente 3 , los cuales tienen mayor peso en las variables de horario de actividad y frecuencia de visita; ambos componentes explican el $66.67 \%$ de la varianza total.

La representación gráfica del ACP manifestó la conformación de 2 grupos que corresponden a las especies diurnas y las nocturnas (Fig. 5).Se observa que las especies con mayor frecuencia de visita, tanto de actividad diurna (C. aura, C. draconoides y C. mexicanus) como nocturna (grupo de murciélagos), se separan más de los grupos principales. Respecto a las especies que se registraron con actividad diurna y nocturna se encontró que $C$. latrans y $U$. cinereoargenteus tienden a alejarse más del grupo central diurno que del nocturno, indicando que es mayor su actividad diurna que la nocturna. Por otra parte, $P$. lotor muestra una mayor dispersión del punto central del grupo nocturno que del diurno, destacando su horario de actividad nocturno.

\section{Discusión}

Cada sitio seleccionado fue diferente en sus características topográficas, lo que genera diferencias en los atributos de la vegetación. El Saucito, con una topografía parecida a un cañón, presenta mayor número de individuos y riqueza de especies vegetales; asimismo, en la Cañada Norte, con características de fondo de cañón, se encontró un alto número de individuos y mayor densidad, por lo que puede considerarse que ese tipo de topografía induce a una mayor abundancia en plantas.

Durante el periodo de estudio, las evidencias visuales en los 4 sitios indicaron que el abastecimiento de agua subterránea fue constante; sin embargo, por medio de los registros fotográficos se verificó que la cantidad de agua aportada varió en el transcurso del día.

La cantidad de agua acumulada en los sitios mostró una alta fluctuación debido a las variaciones diarias y principalmente a los eventos climáticos extremos, como fue el caso del paso de los huracanes Henriette e Ivo por Baja California Sur, en septiembre de 2007. El arrastre de arena, rocas y vegetación, exacerbado por los volúmenes de agua y escurrimientos originados por dichos fenómenos, creó nuevos CAS y otros desaparecieron, o bien, modificaron su forma o cantidad de agua acumulada.

Los arroyos y cañones estudiados son relevantes por las características microambientales que presentan y por la acumulación de agua, que en las zonas áridas es un elemento sumamente importante, entre otros aspectos, porque influye en la distribución de especies animales (JaramilloMonroy et al., 1991; Turner et al., 2004).

De las 41 especies y el grupo de los murciélagos registrados en los CAS, el 59.5\% fueron especies que visitaron 1 o 2 CAS, el $26.2 \%$ visitaron entre 3 y 9 CAS, y el $14.3 \%$ fueron especies que comúnmente usan diferentes CAS, dentro de éstas se encuentran especies de aves y mamíferos.

Los anfibios se caracterizan por estar estrechamente asociados al agua debido a sus adaptaciones biológicas; no obstante, durante este estudio estos organismos no se 
registraron por medio de cámaras-trampa. De las 3 especies nativas registradas para la región (Grismer, 2002), se han observado directamente en los 4 sitios a Anaxyrus punctatus y en El Saucito a Pseudacris hypochondriaca. De igual manera, por observación directa, en El Sauzoso se ha registrado Lithobates sp., una de las especies introducidas en la península de Baja California (Grismer, 2002).

Para la zona de estudio están registradas en la literatura 29 especies de reptiles (Grismer, 2002), un buen número de las cuales por observaciones directas; sin embargo, con el método utilizado en este estudio, sólo se registraron 3 especies; 2 en un solo caso: A. tigris y $U$. nigricaudus. El sitio donde se registraron más individuos del grupo de los reptiles fue El Sauzoso, cuyas características topográficas son de arroyo abierto, lo cual, dadas las necesidades ectotérmicas de los reptiles, hace predecible su encuentro ahí con mayor frecuencia, particularmente de las especies que prefieren sustrato arenoso. Lo anterior se confirma por la mayoría de registros en los espacios abiertos de los 4 sitios muestreados de $C$. draconoides, especie encontrada con frecuencia, asoleándose o forrajeando en este tipo de sustrato.

En comparación con los otros grupos taxonómicos, el de las aves fue el que mayor número de especies presentó El sitio con mayor riqueza específica y abundancia de aves fue El Camarón, con características topográficas entre arroyo y cañón, influenciado principalmente por los palmares que se encuentran a lo largo del arroyo; asimismo, de los 4 sitios, fue el que presentó la mayor altura promedio. La especie que con más frecuencia visitó los CAS fue C. aura, seguida de C. mexicanus.

En el área de estudio se encontraron individuos de especies de aves registradas como migratorias: $A$. macularia (asociada con hábitats acuáticos) y $P$. melanocephalus (Sibley, 2001), sólo en El Sauzoso; Sayornis saya, presente 1 vez en El Camarón y 1 en El Saucito, y M. alcyon, migratoria que con frecuencia se relaciona con CAS dulces, salados y/o salobres (Sibley, 2001), se registró en 1 ocasión en El Saucito.

Especies importantes en el uso de los CAS fueron las aves rapaces, como $B$. jamaicensis, observada dentro de los CAS para bañarse o refrescarse. Esto contradice lo descrito por Cade (1965), quien menciona que la presencia de aves depredadoras en los CAS es principalmente para la caza de su alimento. En general, en los CAS estudiados se registró más del 50\% de las especies registradas para la región (Sibley, 2001).

En el área de estudio se han registrado13 especies de mamíferos medianos y grandes (Álvarez-Castañeda y Patton, 1999, 2000), 6 en los CAS de los 4 sitios. Dentro de los pequeños mamíferos se encuentra $A$. leucurus, registrado en un solo CAS en la Cañada Norte; fue comúnmente observado en áreas alejadas de CAS; posiblemente su rareza cerca de éstos se deba a que su consumo de agua libre es mínimo. En el caso de los murciélagos, en la región se han registrado17 especies clasificadas en 3 familias (ÁlvarezCastañeda y Patton, 1999); sin embargo, dada la dificultad de una identificación precisa de las especies, como ocurrió en el trabajo de O'Brien et al. (2006), todas las observaciones se consideraron como grupo, representando una especie de mamífero. Los murciélagos se presentaron con frecuencia en los CAS de El Sauzoso y El Camarón, sitos con mayor cantidad de agua libre, y estuvieron ausentes en la Cañada Norte, donde la cantidad de agua disponible fue mínima. El Camarón fue el sitio más frecuentado por los mamíferos silvestres, lo cual puede deberse a que en ese sitio existen varias fracturas topográficas que sirven de paso ideal para los movimientos de los animales terrestres.

Adicionalmente, en los 4 sitios estudiados se registraron 3 especies domésticas de las 5 que se conocen para la zona de estudio (Mesa-Zavala, 2008). Varios burros, Equus asinus, fueron observados en 3 sitios, con preferencia en los CAS de El Camarón. A menudo se observó ganado vacuno, Bos taurus, en El Sauzoso y El Saucito, ambos sitios preferidos por los rancheros para llevar su ganado, por la disponibilidad de agua y vegetación forrajera a lo largo del año. El caballo, Equus caballus, se utiliza por los rancheros para arrear ganado; así, su registro fotográfico fue con el jinete o en los CAS cercanos a un rancho.

Ovis canadensis, especie cinegética con protección especial (SEMARNAT, 2010). Se observó usando los CAS en 3 sitios en diferentes ocasiones. En El Camarón fue donde se hizo el mayor número de observaciones, incluyendo las de individuos aislados y rebaños. Su nula presencia en El Saucito podría deberse a que está ubicado en una parte de la sierra constituida por lomeríos con escaso terreno de escape (topografía escabrosa y pendientes mayores a $60^{\circ}$ ), factor fundamental en la conducta antidepredadora de esta especie (López et al., 1999; Krausman y Shackleton, 2000; McKinney et al., 2003; Álvarez-Cárdenas et al., 2009). En este trabajo no se encontraron evidencias de que la cobertura de vegetación pueda considerarse indicadora de la presencia de $O$. canadensis, ya que no fue medida con respecto a la visibilidad que deben tener estos animales (menor a 1.5 $\mathrm{m}$ de altura) (Risenhoover y Bailey, 1985). Otra especie cinegética importante en la región es Odocoileus hemionus; sin embargo, no se registró su presencia durante el estudio, posiblemente porque prefiere hábitats que se encuentran en las planicies o mesetas en elevaciones mayores a la de los sitios muestreados.

Los CAS pueden funcionar como centro de acumulación de especies presa; sin embargo, en este estudio no se observó algún tipo de comportamiento derivado de la interacción presa-depredador, coincidiendo con la investigación de DeStefano et al. (2000), quienes mencionan 
que los depredadores se acercan a las áreas con CAS para tomar agua más que para cazar.

Respecto al uso de los CAS, la mayoría de las especies acuden a estos sitios durante el día, cuando la temperatura es mayor y las necesidades fisiológicas aumentan. Dentro de las especies que se registraron en el horario diurno se encuentra T. taxus, carnívoro con hábitos principalmente nocturnos (Aranda, 2000) que destaca por encontrarse dentro de la categoría de amenazada (SEMARNAT, 2010).

A pesar de que el método de cámara-trampa no reveló la riqueza de especies presentes en la porción sur de la sierra (Mesa-Zavala, 2008), sí permitió registrar las que acuden a los CAS en arroyos intermitentes y su frecuencia de visita, así como identificar el horario de su actividad (diurna y nocturna). Con este método se pudo detectar si las visitas fueron de individuos solitarios, de grupos de la misma especie, como $C$. latrans, $O$. canadensis, $P$. lotor, $U$. cinereoargenteus, $C$. aura, de aves pequeñas y de murciélagos, así como la presencia simultánea de diversas especies, destacando el caso de $C$. aura con $U$. cinereoargenteus. De igual manera, se registró la actividad de especies raras o difíciles de observar, como T. taxus y L. rufus, y se pudo constatar la presencia de especies como O. canadensis en CAS con terreno de escape accesible, lo cual está considerado requisito elemental para su presencia (Wakeling y Miller, 1989; Krausman y Shackleton, 2000; Álvarez-Cárdenas et al., 2001). Se encontró que no hay modificación en el comportamiento de los animales con las cámaras-trampa, ya que se han registrado organismos que con anterioridad estuvieron cerca de estos aparatos, tal y como lo observaron Heilbrun et al. (2003) y Seydack (1984) con recapturas fotográficas.

Por lo anterior, se considera que el uso de las cámarastrampa, en comparación con otros métodos para identificar las especies que utilizan los CAS, es efectivo y no invasivo, en particular para especies esquivas a los humanos, como B. jamaicensis, Corvus corax, L. rufus, O. canadensis, T. taxus, $U$. cinereoargenteus, entre otros. Sin embargo, con este método es difícil la identificación de especies pequeñas (roedores y murciélagos), como lo mencionaron O'Brien et al. (2006); además, como lo describió Karanth (1995), las condiciones climáticas y los problemas técnicos modifican necesariamente el esfuerzo de muestreo.

En Baja California Sur, la mayoría de los ambientes tienen características semiáridas, por lo cual los CAS se convierten en un recurso vital para las comunidades silvestres, destacando especies cinegéticas y de importancia ecológica, como $O$. canadensis, L. rufus, T. taxus, aves residentes y migratorias, algunas especies de reptiles y las frágiles especies nativas de anfibios. De igual forma, los CAS son importantes para la gente local, quienes de forma directa o indirecta (a través del ganado doméstico y otras especies introducidas) utilizan el recurso compitiendo con la fauna silvestre por el recurso y en ocasiones trasforman drásticamente el ambiente. Es por ello necesario realizar esfuerzos de investigación y conservación con enfoque en el manejo sustentable, analizando el efecto de las especies introducidas y valorando la fragilidad del hábitat por la vulnerabilidad de los sitios a los eventos climáticos.

\section{Agradecimientos}

Este trabajo fue realizado en el Centro de Investigaciones Biológicas del Noroeste, S.C. con financiamiento de proyectos institucionales y apoyo becario del Consejo Nacional de Ciencia y Tecnología para los estudios de posgrado de la M. en C. Erika Mesa Zavala. Agradecemos al Dr. Gustavo Arnaud Franco, Dra. Lía Méndez Rodríguez, M. en C. Diana Dorantes Salas y Genaro Gurrola Rocha, por sus valiosos comentarios al manuscrito; al M. en C. Alejandro Ramos Rodríguez, Ing. Joaquín Rivera Rosas y Lic. Reyna Rubí Romero, por su ayuda en el uso de los programas computacionales; a los encargados, propietarios y demás autoridades, por las facilidades brindadas para la realización del estudio y colocación de cámaras-trampa y a Miguel Domínguez, Armando Tejas y Abelino Cota, por su colaboración en el trabajo de campo.

\section{Literatura citada}

Álvarez-Cárdenas, S., I. Guerrero-Cárdenas, S. Díaz, P. Gallina y S. Gallina. 2001. The variables of physical habitat selection by desert bighorn sheep (Ovis canadensis weemsi) in the Sierra del Mechudo, Baja California Sur, México. Journal of Arid Environments 49:357-374.

Álvarez-Cárdenas, S., P. Galina-Tessaro, S. Díaz-Castro, I. Guerrero-Cárdenas, A. Castellanos-Vera y E. Mesa-Zavala. 2009. Evaluación de elementos estructurales del hábitat del borrego cimarrón en la Sierra del Mechudo, Baja California Sur, México. Tropical Conservation Science 2:189-203.

Álvarez-Castañeda, S. T. y J. L. Patton. 1999. Mamíferos del noroeste de México. Centro de Investigaciones Biológicas del Noroeste, S.C., México. 583 p.

Álvarez-Castañeda, S. T. y J. L. Patton. 2000. Mamíferos del noroeste de México. II. Centro de Investigaciones Biológicas del Noroeste, La Paz, Baja California Sur. 873 p.

Aranda, M. 2000. Huellas y otros rastros de los mamíferos grandes y medianos de México. Instituto de Ecología, Xalapa, Veracruz, México. 212 p.

Briones, O. 1994. Origen de los desiertos mexicanos. Ciencia 45:263-279.

Broyles, B. 1995. Desert wildlife water developments: Questioning use in the southwest. Wildlife Society Bulletin 23:663-675.

Broyles, B. y T. L. Cutler. 1999. Effect of surface water on desert 
bighorn sheep in the Cabeza Prieta National wildlife refuge, southwestern Arizona. Wildlife Society Bulletin 27:10821088.

Cade, T. J. 1965. Relations between raptors and columbiform bird at a desert water hole. The Wilson Bulletin 77:340-345.

DeStefano, S., S. L. Schmidt y J. C. deVos. 2000. Observations of predator activity at wildlife water developments in southern Arizona. Journal of Range Management 53:255-258.

García, E. 1973. Modificaciones al sistema de clasificación climática de Koppen: para adaptarlo a las condiciones de la República Mexicana. Instituto de Geografía, UNAM, México, D.F. 243 p.

Grismer, L. L. 2002. Amphibians and reptiles of Baja California: including its Pacific islands and the islands in the Sea of Cortés. University of California Press, Berkeley. 339 p.

Heilbrun, R. D., N. J. Silvy, M. E. Tewes y M. J. Peterson. 2003. Using automatically triggered cameras to individually identify bobcats. Wildlife Society Bulletin 31:748-755.

Jaramillo-Monroy, F., J. Vallejo, C. Sepúlveda y R. Mendoza. 1991. Observaciones sobre la población del borrego cimarrón en el área de Santa Rosalía, Baja California Sur, México. Ecologica 1:22-24.

Kaiser, H. F. 1960. The application of electronic computers to factor analysis. Educational and Psychological Measurement 20:141-151.

Karanth, K. U. 1995. Estimating tiger populations from camera-trap data using capture-recapture models. Biological Conservation 71:333-338.

Krausman, P. R. y D. M. Shackleton. 2000. Bighorn sheep. In Ecology and management of large mammals in North America, S. Demarais y P. R. Krausman (eds.). Prentice-Hall, New Jersey. p. 517-544.

Krausman, P. R., S. S. Rosenstock y J. W. Cain III. 2006. Developed water for wildlife: science, perception, values and controversy. Wildlife Society Bulletin 34:563-569.

León de la Luz, J. L., J. Rebman, M. Domínguez-León y R. Domínguez-Cadena. 2008. The vascular flora and floristic relationships of the sierra de la Giganta in Baja California Sur, México. Revista Mexicana de Biodiversidad 79:29-65.

López, S. E., R. M. Lee, J. C. De Vos, R. E. Schweinsburg y S. G. Luna. 1999. Relación uso-disponibilidad de componentes topográficos, un modelo de calidad del hábitat para el borrego cimarrón, en Sonora, México. Acta Zoologica Mexicana 76:17-34.

MacMahon, J. A. 1979. North American deserts: their floral and faunal components. In Arid land ecosystems: structure, funtioning and management, D. W. Goodall, R. A. Perry y R. L. Simpson (eds.). Cambridge University Press. p. 21-82.

Marshal, J. P., V. C. Bleich, P. R. Krausman, M. L. Reed y N. G. Andrew. 2006. Factors affecting habitat use and distribution of desert mule deer in an arid environment. Wildlife Society Bulletin 34:609-619.
McKinney, T., S. R. Boe y J. C. De Vos. 2003. Gis-based evaluation of escape terrain and desert bighorn sheep populations in Arizona. Wildlife Society Bulletin 31:1229-1236.

Mesa-Zavala, E. 2008. Caracterización del hábitat y fauna asociada a los cuerpos de agua superficial en el sur de la Sierra de El Mechudo, B.C.S. México. Tesis, Centro de Investigaciones Biológicas del Noroeste, La Paz, Baja California Sur. 90 p.

Nagy, K. A. 1975. Water and energy budgets of free-living animals: Measurement using isotopically labeled water. In Environmental physiology of desert organisms, N. F. Hadley (ed.). Dowden, Hutchinson and Ross, Stroudsburg, Pennsylvania. p. 227-245.

O’Brien, C. S., R. B. Waddell, S. S. Rosenstock y M. J. Rabe. 2006. Wildlife use of water catchments in Southwestern Arizona. Wildlife Society Bulletin 34:582-591.

Polis, G. A.1994. The ecology of desert communities.University of Arizona Press, Tucson. 528 p.

Risenhoover, K. L. y J. A. Bailey. 1985. Foraging ecology of mountain sheep: implications for habitat management. Journal of Wildlife Management 49:797-804.

Rosenstock, S. S., V. C. Bleich, M. J. Rabe y C. Reggiardo. 2005. Water quality at wildlife water sources in the Sonoran desert, United States. Rangeland Ecology and Management 58:623627.

Rzedowski, J. 1978. Vegetación de México. Limusa, México, D. F. 432 p.

SEMARNAT (Secretaría del Medio Ambiente y Recursos Naturales). 2010. Norma Oficial Mexicana-059. Especies protegidas en peligro, amenazadas, o en extinción. Diario Oficial de la Federación, 30 de diciembre.

Seydack, A. H. W. 1984. Application of a photo-recording device in the census of larger rain-forest mammals. South African Journal of Wildlife Research 14:10-14.

Shmida, A., M. Evenari y I. Noy- Meir. 1986. Hot desert ecosystems: an integrated view. In Hot deserts and arid shrublands, M. Evenari, I. Noy- Meir y D. W. Goodall (eds.). Elsevier, Amsterdam. p. 379-387.

Sibley, A. D. 2001. The Sibley guide to birds. National Audubon Society, New York. 545 p.

Turner, J. C. y R. Weaver. 1980. Water. In The desert bighorn, its life history, ecology and management, G. Monson and. L. Sumner (eds.). University of Arizona Press, Tucson, Arizona. p.100-112.

Turner, J. C., C. L. Douglas, C. R. Hallum, P. R. Krausman y R. R. Ramey. 2004. Determination of critical habitat for the endangered nelson's bighorn sheep in Southern California. Wildlife Society Bulletin 32:427-448.

Wakeling, F. B. y W.H. Miller. 1989. Bed site characteristic of desert bighorn sheep in the Superstition Mountains, Arizona. Desert Bighorn Council Transactions 33:6-8.

Whitford, W. 2002. Ecology of desert systems. Academic, New York. 343 p. 